\title{
Vollvirtualisierung - Beitrag der Wirtschaftsinformatik zu einer Vision
}

\author{
DOI 10.1007/s11576-008-0129-7
}

\section{Die Autoren}

Prof. Dr. Hans Ulrich Buhl

Lehrstuhl für BWL, Wirtschafts-

informatik, Informations- und

Finanzmanagement

Universität Augsburg

Universitätsstraße 16

86135 Augsburg

Deutschland

hans-ulrich.buhl@wiwi.uni-augsburg.de

Prof. Dr. Robert Winter

Institut für Wirtschaftsinformatik

Universität St. Gallen

Müller-Friedberg-Strasse 8

9000 St. Gallen

Schweiz

robert.winter@unisg.ch

This article is also available in English via http://www.springerlink.com and http://www.bise-journal.org: Buhl HU, Winter R (2008) Full Virtualization - BISE's Contribution to a Vision. Bus Inf Syst Eng. doi: 10.1007/s12599-0080023-2.
Es gibt Innovationen, deren lange Erfolgsgeschichte im Laufe der Zeit in den Hintergrund gerät und die erst Jahrzehnte später durch das Marketing einiger Unternehmen wieder aufgefrischt ins Rampenlicht treten. Im Bereich der Informations- und Kommunikationstechnik (IKT) erfährt zurzeit das Thema Virtualisierung diese Renaissance. Schon bereits Ende der 1960er-Jahre wurde in der laut IBM wichtigsten Produktankündigung der Unternehmensgeschichte, der Systemfamilie /360, erstmalig in einem Großrechner Virtualisierungstechnik serienreif zum Einsatz gebracht. Auf einem einzelnen System konnten mehrere Benutzer gleichzeitig über unabhängig voneinander arbeitende virtuelle Maschinen verfügen und so den Bedarf an zusätzlicher und teurer Hardware reduzieren. Dieser technische Fortschritt war nicht nur für IBM, sondern auch für etliche andere Unternehmen ein wichtiger Grundstein für deren langjährige Erfolgsgeschichte. So wie damals sind es auch heute die ökonomischen Faktoren, die dem Thema Virtualisierung wieder viel Aufmerksamkeit zukommen lassen. Getreu dem Motto „Back to the Roots“ initiiert die aktuelle Neuauflage bei der IKT eine Trendumkehr von vielen dezentralen wieder zurück zu zentralen und leistungsstarken Großsystemen, um dadurch die Anschaffungs-, Betriebs- und Wartungsaufwände zu reduzieren. Dass man sich vor über vierzig Jahren mit Virtualisierung nicht nur auf infrastruktureller Ebene beschäftigte, sondern es zumindest auch Visionen einer Anwendung gab, die das Leben des Menschen verändern sollte, zeigt Stanislav Lem in seinem 1964 erschienenen Hauptwerk "Summa technologiae“. Dort skizziert er eine "phantomatische Maschine“, die es daran angeschlossenen Menschen ermöglicht, fiktive Situationen, wie z. B. einen Ballonflug, zu sehen und zu erleben. Heute werden diese real gewordenen „Maschinen“ mit "Cyberspace ", „virtuelle Welt" oder "Virtual Reality" bezeichnet und als Simulationssysteme z. B. bei der Pilotenausbildung oder den Massive-Multiplayer-Online-Games, wie z. B. Second Life, mit Tausenden von Nutzern eingesetzt.

Trotz der unverkennbaren Parallelen hat sich im Vergleich zur damaligen Ausgangssituation dennoch etwas geändert. Virtualisierung, wie sie heute umgesetzt wird und für morgen geplant ist, hat die infrastrukturelle Ebene längst verlassen und ist weit in die wirtschaftlichen und sozialen Strukturen unserer Gesellschaft eingedrungen. Für die Wirtschaftsinformatik als Wissenschaft eröffnet sich dadurch eine Reihe von Themen mit klarem und spannendem Bezug auf Geschäftsmodelle, Geschäftsprozesse und deren abgestimmtes Zusammenspiel mit Anwendungssystemen und Infrastruktur. Darüber hinaus verändert Virtualisierung aber auch das Verhältnis zwischen Mensch und Maschine und hat damit zunehmende Auswirkungen auf den Einzelnen und die soziale Gemeinschaft. Wohin würde dieser Trend führen, wenn wir ihn kontinuierlich vorantreiben und alle Möglichkeiten ausschöpfen? Genau diese aus Sicht der Wirtschaftsinformatik interessanten Themen und Fragestellungen versuchen wir, im Rahmen dieses Schwerpunktthemas näher zu beleuchten.

Doch was ist Virtualisierung?

Laut Duden hat "virtuell" seinen ethymologischen Ursprung im lateinischen Wort für "Tugend", "Kraft" und „Vermögen" und bedeutet "was nach Anlage oder Vermögen der Möglichkeit nach vorhanden ist". "Virtuell" entspricht der Eigenschaft einer nicht vorhandenen Entität, in Form und Wirkung einer realen Entität gleichgestellt zu sein. Virtualisierung ist in einer ersten Näherung somit die Abbildung vorhandener Strukturen oder die Erschaffung neuer Strukturen, wobei Form und Wirkung i. d. R. mit Hilfe von Informations- und Kommunikationssystemen (IKS) erzeugt werden. Für die Wirtschaftsinformatik bedeutet dies vor allem den ökonomisch sinnvollen Handlungsbedarf zu erkennen und die für die Transformation notwendigen Voraussetzungen zu schaffen. Gemäß dem Ansatz „think big, start small“ werden mit 
dem angestrebten Ziel vor Augen so Schritt für Schritt Lösungen entwickelt, die wieder neu zu bewertende Möglichkeiten eröffnen.

Die Vision der Vollvirtualisierung, die eine durchgängige Abbildung sämtlicher Strukturen unseres Lebens durch IKS anstrebt, könnte ein mögliches Ziel einer konsequenten Weiterentwicklung sein. Was wären die Implikationen dieser Vision auf Wirtschaft, Gesellschaft und Individuen, würde man sie als Grenzfall einer Vision "vollständig" zu Ende denken?

Vermutlich würden zunächst im Bereich der Wirtschaft sämtliche am inner- und außerbetrieblichen Leistungsprozess beteiligten Produkte und Dienstleistungen, die aufgrund der Vollvirtualisierung keinen Nutzen mehr haben, in eine Nische abge-

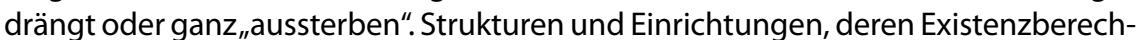
tigung in der physischen Präsenz dieser Güter bestand, müssten sich neu ausrichten und anderen Aufgaben widmen. Ein prägnantes Beispiel dafür sind der Briefverkehr oder die betriebliche Hauspost, die in großen Teilen durch das Medium E-Mail abgelöst wurden und im Zuge der Einführung rechtsgültiger elektronischer Unterschriften noch weiter an Bedeutung verlieren dürften. Ähnliches gilt für Zeitungen und viele andere Printmedien mit allen - durchaus auch problematischen Folgen.

Auf der anderen Seite würden Produkte zunächst virtuell entwickelt und auf ihr Marktpotenzial getestet werden, bevor diese in die reale Produktion gehen. Dieser Umbruch im Zusammenspiel zwischen virtuellen und physischen Gütern und Prozessen hätte Einfluss auf den gesamten Wertschöpfungsprozess. Lieferanten und Abnehmer wären gemeinsam in einem virtuellen Entwicklungs- und Produktionsprozess integriert und könnten so Kosten und Risiken reduzieren. Die Abdeckung komplexer Anforderungen erfolgt durch neue Geschäfts- und Preismodelle, die durch Virtualisierung einen flexiblen und bedarfsorientierten Produkt- und Leistungsbezug ermöglichen. Dies gilt mehr und mehr auch in traditionell ,physischen“ Branchen. So zeigen z. B. in der Bauwirtschaft Beschaffungsmodelle wie "Löcher statt Bohrmaschinen“ oder "Druckluft statt Kompressoren", dass anstelle physischer Maschinen deren Leistungen mit zugesicherter Qualität und Verfügbarkeit "on Demand" angeboten und nachgefragt werden können. Eine ähnliche Veränderung wird es für Dienst- und Beratungsleistungen geben, die aufgrund von Virtualisierung nicht mehr vor Ort stattfinden müssen und so überall und jederzeit durch den Kunden in Anspruch genommen werden können. Meetings und Konferenzen, die in virtuellen Räumen abgehalten werden, bieten dem Veranstalter und Teilnehmern nicht nur maximale Flexibilität, sondern vermeiden ebenfalls kosten-, zeit- und umweltintensive Anfahrtswege. Einige gesellschaftliche Einrichtungen, wie Universitäten oder Behörden könnten so in bestimmten Bereichen durch IKT ersetzt werden, die sich wiederum an wenigen zentralen Standorten aggregieren lässt. Im anderen Fall, bei dem das Realprodukt dem virtuellen folgt, ließen sich mögliche Entwicklungs- und Absatzrisiken bereits im Vorfeld identifizieren und überprüfen. Die Komplexität dieser virtuellen Produkt- und Leistungserstellung führt im B2B-Geschäft allerdings auch zu neuen organisatorischen Vernetzungsstrukturen und zunehmenden Abhängigkeiten in der Wertschöpfungskette. Die dadurch entstehenden Risiken lassen sich nur schwer abschätzen und können, wie das Beispiel der Finanzkrise zeigt, einen unkontrollierten Flächenbrand auslösen.

Eine Vollvirtualisierung würde aber nicht nur wirtschaftliche Strukturen vollkommen verändern, sondern auch die Gesellschaft und die Individuen selbst. Gerade die so vorteilhafte Eigenschaft der Zeit- und Ortsungebundenheit bei der Erstellung und beim Konsum virtueller Substitute führt möglicherweise bei Vielen zu einer verstärkten sozialen Isolation, da überwiegende Teile der Arbeitszeit und Freizeit ausschließlich zu Hause verbracht werden können. Der direkte Sozialkontakt würde sich hauptsächlich auf die im gleichen Haushalt lebenden Personen beschränken, alles andere findet in der Online-Community statt. Es lässt sich bezweifeln, dass virtuelle Beziehungsformen, wie sie derzeit möglich sind und ausgelebt werden, langfristig einen adäquaten Ersatz bieten können, um soziale Defizite zu kompensieren.

Ist dieses Szenario trotz der positiven Seiten wirklich erstrebenswert? Wie können die negativen Effekte einer Vollvirtualisierung vermieden werden? Die Aufgabe der Wirtschaftsinformatik ist, proaktiv zu handeln und sich den relevanten Fragen zu stellen:Welche Fortschritte im Bereich der Virtualisierung auf den einzelnen IKS-Ebenen 
gibt es? Welcher weitere Handlungs- und Forschungsbedarf besteht und bis zu welchem Grad sollen Wissenschaft und Praxis die Vision der Vollvirtualisierung weiterverfolgen?

In den letzten Jahren konnten sich etliche erfolgreiche Geschäftsmodelle etablieren, die hauptsächlich auf Virtualisierung setzen. Die Grundidee der meisten davon basiert auf der schnellen und flexiblen Zusammenführung von Akteuren in einem virtuellen Ort, einem Prozess oder einer Gemeinschaft. Wertschöpfungsnetze lassen sich dadurch global optimieren, indem sehr viele und günstige Standorte, Anbieter oder Produkte integriert werden und die Anzahl an Kunden nahezu beliebig groß werden kann. Sehr erfolgreich sind in diesem Umfeld z. B. die großen E-CommerceHandelsplattformen. Andere Anbieter stellen virtuelle Räumen (Virtual Meeting Rooms) zur Verfügung, in denen sich Experten zusammen finden können, um auf Basis von dort verfügbaren 3-D-Modellen oder Simulationen planungsrelevante Entscheidungen zu treffen. Gerade bei großen Projekten können so bereits frühzeitig kostspielige Fehler erkannt und vermieden werden. Militärs setzen diese Technik ein, um taktische Maßnahmen und mögliche Gegenreaktionen in beliebigen Varianten zu simulieren. Für geplante Bauvorhaben werden bspw. optische Eindrücke generiert sowie Funktionalitäten und Strukturen interaktiv überprüft. Durch eine computergestützte visuelle Erweiterung der Realwelt (Mixed Reality), lassen sich zudem die Anforderungen komplexer Vorgänge, wie dies z. B. bei Echtzeitsimulationen in der Medizin notwendig ist, erfüllen.

Virtualisierung ist aber nicht ausschließlich eine Domäne der New Economy und beschränkt sich, wie die Beispiele aus der Baubranche zeigen, nicht mehr auf den Bereich elektronischer Marktplätze, Computersimulationen und Dienstleistungen! Auch viele traditionelle Industrieunternehmen haben erkannt, dass sie dadurch ihre Wettbewerbsfähigkeit nicht nur erhalten, sondern nachhaltig ausbauen können. Der Einfluss von Märkten, Lieferanten, Kunden, Kooperationspartnern und dem Verhalten von Konkurrenten auf die eigene Ertrags- und Risikoposition lässt sich durch entsprechende Simulationen besser vorhersehen. Unternehmen können auf Basis der gewonnen Daten steuernd eingreifen und bspw. die Sourcingstrategie entsprechend anpassen bzw. so robust gestalten, dass mögliche Änderungen zu keinen anderen Entscheidungen führen. Prozesse, die durch Virtualisierung flexibler und effizienter gestaltet werden können, führen zu Kosteneinsparungen und eröffnen neue Möglichkeiten innerhalb der Wertschöpfungskette. Prozessschritte, die sich automatisch der Umweltsituation anpassen, werden dynamisch zu einem Gesamtprozess kombiniert, ohne dass strukturelle Veränderungen im Unternehmen notwendig sind. Der Produktionsfaktor Mensch, der durch diese Transformation an vielen operativen bzw. fertigenden Aktivitäten immer mehr an Bedeutung verliert, kann dadurch seinen Tätigkeitsschwerpunkt auf kreative und nicht automatisierbare Aufgaben mit komplexem Anforderungsprofil verlagern. Auf der anderen Seite wurde aber auch erkannt, dass die unternehmensweite Optimierung des interpersonellen Austauschs impliziten Wissens auf die Unternehmensprozesse einen entscheidenden Einfluss hat. Mit Unterstützung von IKT werden virtuelle Expertenteams gebildet und vorhandene, meist informelle Wissensnetzwerke problemspezifisch analysiert und anschließend zielgerichtet ausgeschöpft.

Darüber hinaus ist Virtualisierung immer noch ein Thema der IKT-Infrastruktur selbst. Hier gilt: IT virtualisiert IT. Die IKT-Infrastruktur leistet die Berechnungsarbeit, sorgt für den Datentransport und stellt notwendigen Speicherplatz zur Verfügung. Eine Kapazitätsbeschränkung auf das jeweilige physisch vorhandene Einzelsystem wird durch Virtualisierung weitestgehend aufgehoben, indem viele Rechner- und Speichersysteme zu einem effizienten Netzverbund (Grid) aggregiert werden. Unternehmen mit einer sehr leistungsstarken IKT-Infrastruktur stellen deren Kapazität inner- und außerbetrieblichen Leistungsabnehmern als sogenannte "Wolke“ für die Ausführung von Applikationen bereit (Cloud-Computing), so dass für diese keine eigene Infrastruktur betrieben oder weiter ausgebaut werden muss. Die Anzahl der Cloud-Anbieter wird sich nach Meinung von Steve Balmer, CEO bei Microsoft, in den kommenden Jahren auf wenige marktbeherrschende Anbieter reduzieren, zu denen er in erster Linie Amazon, Google, IBM und Microsoft zählt. Diese quasi beliebig skalierbaren Rechenkapazitäten bilden die Grundvoraussetzung für zukünftig noch komplexere, betriebswirtschaftliche Virtualisierungsanwendungen, die von der ei- 
genen Systemlandschaft wirtschaftlich nicht bewältigt werden können. Unternehmen, die weiterhin eine eigene IKT-Infrastruktur betreiben, können ihrerseits durch Server- und Desktopvirtualisierung die Anzahl notwendiger Hardwaresysteme reduzieren und so enorme Kosteneinsparungen für Anschaffung und Betrieb realisieren. Hier geht Gartner in einer aktuellen Studie davon aus, dass dieser Konsolidierungstrend bis zum Jahr 2012 den größten Einfluss auf die Daten- und Rechenzentren haben wird.

Es wird deutlich, dass wir der Vision der Vollvirtualisierung in vielen Bereichen bereits näher gekommen sind. Wesentliche Treiber sind ökonomische Faktoren, die den Transformationsaufwand rechtfertigen und Unternehmen dazu veranlassen, hier Investitionen zu tätigen. Die Grenzen, die meist von den technischen Möglichkeiten vorgegeben werden, verschieben sich durch die voranschreitende Entwicklung der Computerleistung, der Vernetzung und der verfügbaren Bandbreiten. Globale Netzwerke verbinden potenziell viele Teilnehmer und ermöglichen dadurch mehr Offenheit und Toleranz. Eine zunehmende Technisierung und Vernetzung führt aber auch zu Abhängigkeiten. Die Gesellschaft und die Individuen werden sich dieser Situation anpassen und entsprechende Veränderungen erfahren. Die Herausforderung wird sein, die mit der Vision verbundenen Chancen und Möglichkeiten im Hinblick auf die gleichzeitig vorhandenen Risiken und Gefahren optimal auszubalancieren.

Dieses Schwerpunktthema umfasst ausgewählte Beiträge, die sich verschiedenen Teilaspekten widmen. Drei Artikel in der Rubrik WI - Aufsatz präsentieren neue Forschungsergebnisse. Kai Fischbach, Peter A. Gloor und Detlef Schoder stellen einen Ansatz zur Analyse informeller Kommunikationsnetzwerke vor. Kai Riemer und Stefanie Filius widmen sich dem Thema der richtigen Medienwahl in virtualisierten Arbeitskontexten und zeigen, welches Verbesserungspotenzial beim Medieneinsatz in verteilten Teams besteht. Stephan Aier und Robert Winter schlagen eine virtuelle Architektur vor, die durch Entkopplung von IT und Business den Anforderungen an Änderbarkeit in beiden Ebenen besser gerecht wird. Gegenstand des Beitrags von Jens Vykoukal, Martin Wolf und Roman Beck in der RubrikWI - State of the Art ist das wachsende Angebot an Grid-Services, die Unternehmen on-demand in den Wertschöpfungsprozess einbinden können, um nicht nur Kosten zu sparen, sondern auch flexibel auf Veränderungen reagieren zu können. Neben diesen wissenschaftlichen Beiträgen beinhaltet dieses Heft ein WI - Schlagwort zum Thema „Virtuelle Welten“ von Danny Pannicke und Rüdiger Zarnekow sowie ein WI - Interview mit Dr. Martin Enderle, CEO der Scout24 Holding GmbH, zum Thema „Möglichkeiten und Grenzen der Virtualisierung von Online-Marktplätzen“. Abschließend diskutieren in der RubrikWI - Meinung/Dialog Wissenschaftler aus Wirtschaftsinformatik und angrenzenden Disziplinen das Thema „Was ist eigentlich Grundlagenforschung in der Wirtschaftsinformatik?".

Wir hoffen, durch dieses Schwerpunktthema interessante Einblicke in das Thema „Vollvirtualisierung - Beitrag der Wirtschaftsinformatik zu einer Vision“ gegeben zu haben. Das Thema hat sich über alle IKS-Ebenen hinweg fest etabliert und bietet noch viel Weiterentwicklungspotenzial. Beim Vorantreiben der Vision sollten Wissenschaft und Praxis neben den wirtschaftlichen Gesichtspunkten den Nutzen für den Menschen nicht aus den Augen verlieren.

Prof. Dr. Hans Ulrich Buhl Prof. Dr. Robert Winter 